\title{
Yoga unterstützt die Heilung
}

\author{
Studie mit Frauen nach Brustkrebsoperationen - Kann ein Yoga-Kurs helfen, mit \\ der Diagnose Brustkrebs und der Therapie umgehen zu lernen? Welche Wirkungen hat er? \\ Wie sollte er aufgebaut sein? Diese Fragen werden gegenwärtig im Rahmen einer Studie \\ untersucht, die gemeinsam vom Brustzentrum des Klinikums Berlin Westend und der Alice \\ Salomon-Hochschule durchgeführt wird.
}

$\boldsymbol{\nabla}$ Die Diagnose Brustkrebs trifft die meisten Frauen überraschend. Gleich danach tritt eine Therapie in Kraft, der äußerst belastend ist. Diese Belastungen abzumildern und die Frauen zu unterstützen, sich körperlich zu erholen und psychisch zu stabilisieren, ist die Idee eines Yogakurses für Frauen nach Brustkrebsoperation.

\section{Hintergrund der Studie}

Um heraus zu finden, wie Yoga wirkt und wann der richtige Zeitpunkt zum Üben ist, werden alle Frauen, die sich zum Yogakurs melden, in zwei Gruppen aufgeteilt: Die Interventionsgruppe und die Wartegruppe. Die erste Gruppe startet schon zwei bis drei Tage nach der Operation. Diese Frauen kommen sogar mit Redonbeuteln zu ihrer ersten Yogastunde. Die Wartegruppe beginnt zeitlich versetzt nach fünf Wochen. Eine Psychologin und zwei Ärztinnen helfen bei der Rekrutierung der Frauen. Die Zufallsaufteilung in zwei Gruppen wird durch das Institut für Biometrie und klinische Epidemiologie der Charité supervidiert. Zu Beginn und am Ende des zehnmaligen Übens füllen die Frauen einen standardisierten Fragebogen aus. Eine Doktorandin nimmt die Daten entgegen. Sie wird sie im Rahmen ihrer Dissertation auswerten.

\section{Das Training}

Zweimal wöchentlich findet der YogaKurs statt. Im Wechsel mit einer zweiten Yogalehrerin, die selbst vor 15 Jahren an Brustkrebs erkrankt war, leite ich die Gruppe an. Wir machen kräftigende und entspannende Übungen und achten darauf, dass jede Frau ihren eigenen optimalen Schwierigkeitsgrad bei den Übungen findet. Wer sich beim Üben wohl fühlt, übt mit der richtigen Intensität. Die Frauen sollen ihre körperlichen Fähigkeiten wiedergewinnen, aber auch die Freude an sich selbst. Denn die körperliche Fitness zu verbessern, ist die eine gute Seite, die Yoga hat. Doch auch psychisch wirken die gemeinsamen Übungsstunden positiv. Yoga schult die Konzentration und Achtsamkeit. Durch regelmäßiges Üben können die Frauen innere Ruhe und Kraft finden. Sie lernen, ihre eigenen Bedürfnisse besser einzuschätzen. Auf diese Weise stärken sie sich, um ihre Erkrankung zu überwinden, aber auch um ihren persönlichen Weg durch alle Tipps und Ratschläge zu finden.

Die Yoga-Übungsstunde beginnt in der Rückenlage. Körper, Arme und Beine werden am Boden mobilisiert. Diese Übungen auf der Matte fördern Dehnfähigkeit und Kraft und bieten gleichzeitig maximale Sicherheit und ein verlässliches Maß für die korrekte Körperhaltung. Daran schließen sich vielfältige Atemübungen an. Sie vermitteln ein Gefühl für die Dehnung der Atemräume. Die Atmung wird gleichmäßiger und tiefer. Darüber hinaus werden die Frauen aber auch konzentrierter, achtsamer und spüren, wie die Bewegungen wirken und welche Bewegungen gut tun. Atemübungen erlauben aber auch, „Dampf“ abzulassen und kraftvoll auszuatmen. Übungen im Stand und aus dem Sitz schulen die Beweglichkeit der Arme und
Schultern, kräftigen die Beine und verbessern die Balance. Als Ausklang der Stunde gibt es geführte Konzentrationsübungen. Noch unnötig gehaltene Spannungen werden so abgebaut.

\section{Fazit}

Frauen können die Zeit nach ihrer Operation und den eventuell notwendigen Weiterbehandlungen nutzen, um ihr körperliches und psychisches Wohlergehen durch regelmäßige Yoga-Übungen zu verbessern. Mit Hilfe von Yoga gewinnen sie ihre normale Beweglichkeit und Kraft schneller wieder. Schonhaltungen, die sie sich nach der Operation unbewusst angeeignet haben, werden durch die Übungen überwunden. Ziel ist es, den Frauen ein sicheres Körpergefühl zu vermitteln, damit sie die Übungen am Ende so durchführen und abwandeln, dass sie optimal zu ihnen passen.

Die Teilnahme an der Studie ist rege. Viele Frauen bleiben noch nach Ablauf des zehnmaligen Übens in der Gruppe. Wir freuen uns über die positiven Rückmeldungen, können die subjektiv wahrgenommenen Verbesserungen durch Yoga sehen und hoffen, dass sich die Wirkungen auch mit den Mitteln der empirischen Forschung nachweisen lassen. -

Prof. Dr. Ingrid Kollak

- Alice-Salomon-FH Alice-Salomon-Platz 5 12627 Berlin

- Email:kollak@asfh-berlin.de 